\title{
Analyst
}

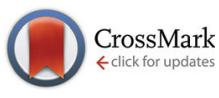

Cite this: DOI: 10.1039/c5an02276b

Received 3rd November 2015,

Accepted 17th February 2016

DOI: $10.1039 / c 5 a n 02276 b$

www.rsc.org/analyst

\section{A highly sensitive colorimetric metalloimmunoassay based on copper-mediated etching of gold nanorods $\uparrow$}

\author{
Fangbin Cheng, ${ }^{a}$ Zhaopeng Chen, ${ }^{{ }^{\mathrm{b}}}$ Zhiyang Zhang ${ }^{\mathrm{b}}$ and Lingxin Chen ${ }^{\mathrm{b}, \mathrm{c}}$
}

\begin{abstract}
A highly sensitive colorimetric metalloimmunoassay with a detection limit of $0.15 \mathrm{ng} \mathrm{ml}^{-1}$ for human IgG based on coppermediated etching of gold nanorods was proposed. The assay is more sensitive than traditional ELISA, electrochemical metalloimmunoassay and HRP mimic nanomaterial tag-based immunoassay.
\end{abstract}

Ultrasensitive sensing of biomolecules and metal ions, such as biomarkers, dopamine, cysteine, $\mathrm{Cu}^{2+}, \mathrm{Hg}^{2+}, \mathrm{Cd}^{2+}, \mathrm{Pb}^{2+}$, etc. plays a very important role in diverse areas, including clinical diagnosis, food control and environmental monitoring. ${ }^{1-7}$ For this purpose, many analytical techniques have been developed, including nonmaterial-based colorimetry and fluorometry, paper-based fluorometry, electrochemistry, atomic spectrometry, immunoassay, etc. Among these, immunoassay was proved to be an effective and ultimately successful method for the quantification of target analytes. ${ }^{8-11}$ Due to its long history, a much more mature operation process and plenty of encouraging experimental results, enzyme-linked immunosorbent assay (ELISA) remains the most popular clinical detection approach. ${ }^{12-15}$ However, ELISA still suffers from the inherent shortcomings of antigen/antibody-protein conjugates, such as easy denaturation and digestion by proteases, the cumbersome production process and time-critical storage life. ${ }^{16-18}$ To overcome these shortcomings, much efforts have been focused on discovering better labels to conjugate antigens or antibodies. ${ }^{16,19-22}$ Due to their unique physical and biochemical properties, metal nanomaterials have been proven to be effective tags in indicating the reaction between antigens and antibodies. Normally, metal nanomaterial tags are quantified by electrochemistry, chemiluminescence, etc. ${ }^{23-27}$

\footnotetext{
${ }^{a}$ Ocean School, Yantai University, Yantai 264005, China

${ }^{b}$ Key Laboratory of Coastal Environmental Processes and Ecological Remediation, Yantai Institute of Coastal Zone Research, Chinese Academy of Sciences, Yantai 264003, China. E-mail: zhpchen@yic.ac.cn; Fax: +86-535 2109133; Tel: +865352109133

${ }^{c}$ College of Chemistry and Chemical Engineering, Yantai University, Yantai 264005, China

$\dagger$ Electronic supplementary information (ESI) available: Experimental details and Fig. S1-S3. See DOI: 10.1039/c5an02276b
}

Although these quantitative measurements provide some advantages, such as low sample volume, excellent sensitivity and relatively inexpensive instrumentation, the promotion and application of these technologies are constrained by their complicated procedures and rigorous experimental details. Spectrophotometric immunoassay remains in the dominant position because most hospitals and disease surveillance organizations, even in remote poor areas or countries, possess microplate readers and spectrophotometers rather than electrochemical or chemiluminescence instruments. So it is necessary to develop effective methods that can sensitively read nanomaterial tags using microplate readers or spectrophotometers. For this purpose, many HRP mimic nanomaterial tags, such as graphene, Au@Pt, Pt@SiO ${ }_{2}$ and platinum nanoparticles, have been applied to immunoassays. ${ }^{16,28-36}$ These methods have also obtained high sensitivity that are comparable to ELISA due to the high catalytic activity of the HRP mimics.

Methods based on catalytic etching of gold nanoparticles have been proven to be simple and sensitive for colorimetric detection of many target analytes, including $\mathrm{Pb}^{2+}, \mathrm{Cu}^{2+}, \mathrm{Co}^{2+}$, $\mathrm{MoO}_{4}{ }^{2-}$, etc. ${ }^{37-40}$ In this work, we combine the conventional metalloimmunoassay with copper-mediated etching of gold nanoparticles proposed in our previous work to develop an effective immunoassay using a microplate reader or a spectrophotometer as readout. The experiment results show that this immunoassay is more sensitive than the traditional ELISA and HRP mimic nanomaterial tag-based immunoassay. ${ }^{31,34,41,42}$

Scheme 1 outlines the procedure of the colorimetric metalloimmunoassay based on copper-mediated etching of gold nanorods using human IgG as the model antigen. Goat anti-human IgG was first adsorbed onto a polystyrene microplate physically. After a conventional sandwich immunoreaction, different amounts of CuS nanocrystals labelled goat antihuman IgG proportional to the target analyte (human IgG) was captured onto the microplate (TEM image shows that the spherical CuS nanocrystals are about $7 \mathrm{~nm}$ and the X-ray diffraction pattern indicates that the synthesized CuS nanocrystals are of high quality. Fig. S1 and S2 in the ESI $\dagger$ ). The cupric ion $\left(\mathrm{Cu}^{2+}\right)$ was then released by the addition of hydrobromic 


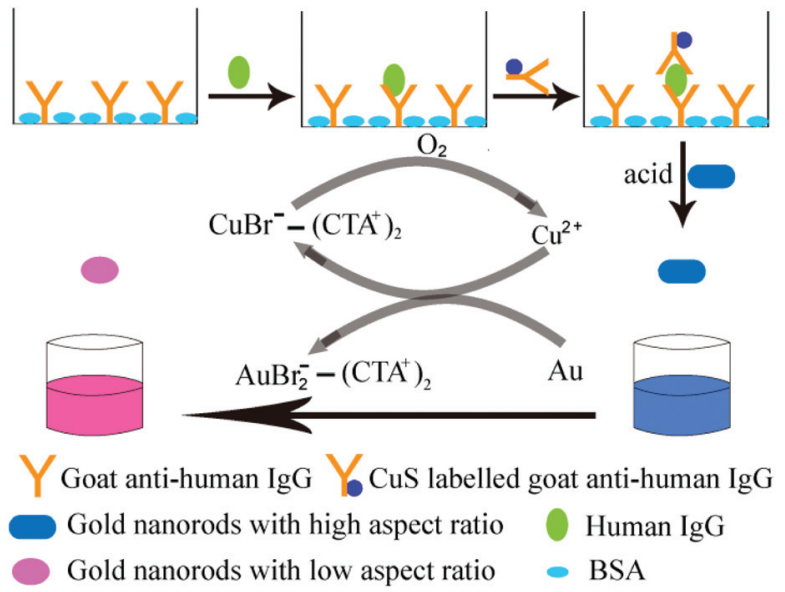

Scheme 1 The schematic illustration of the procedure of the colorimetric metalloimmunoassay.

acid and was determined based on the catalytic etching of gold nanorods.

Fig. 1A shows the changes of the LSPR absorption (A), and color (inset) of gold nanorods induced by different concentrations of $\mathrm{Cu}^{2+}$ which comes from the captured CuS nanocrystals in the sandwich immunoreaction. The longitudinal LSPR absorption peaks were located at 668, 596 and $555 \mathrm{~nm}$ respectively after the incubation of gold nanorods with different concentrations of human IgG-mediated $\mathrm{Cu}^{2+}$. The solution of gold nanorods also changed from blue to purple and then to red with the increase of the concentration of human IgG. The

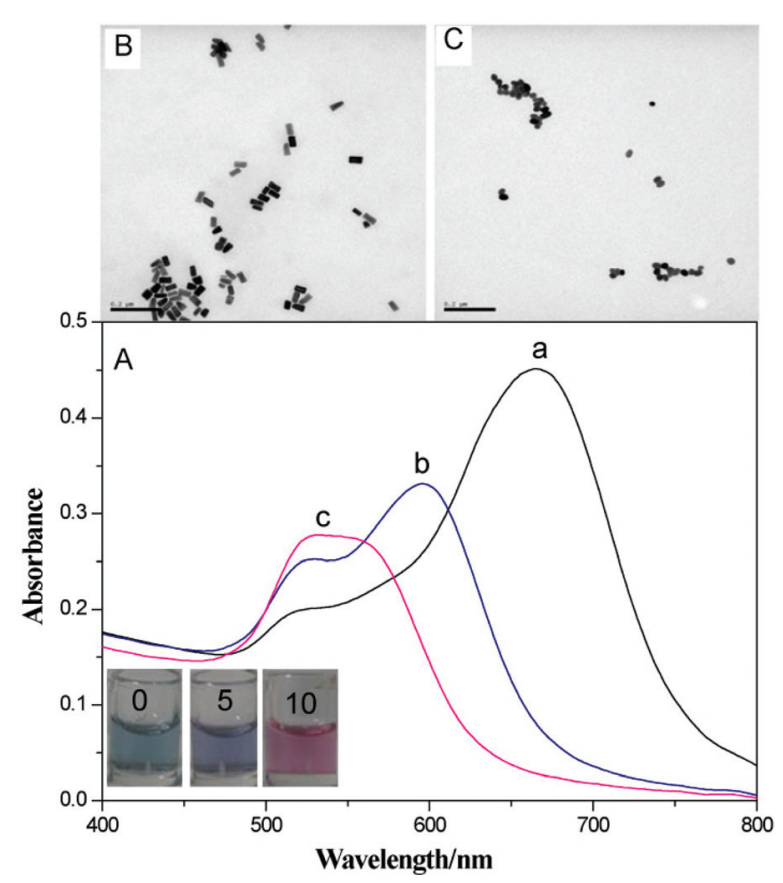

Fig. 1 LSPR absorption spectra (A) and the colors (inset) corresponding to 0 (a), 5 (b) and $10 \mathrm{ng} \mathrm{ml}^{-1}$ (c) human IgG. $B$ and $C$ are the TEM images of gold nanorods corresponding to 0 (B) and $10 \mathrm{ng} \mathrm{ml}^{-1}$ (C) human IgG. peak-shift and color change are attributed to the anisotropic corrosion of gold nanorods along the longitudinal direction due to the less surface passivation and/or the higher reaction activities at the tips (Fig. 1B and C). ${ }^{43}$ Obviously, the peak-shift and color change can be applied to indicate the target analyte concentration.

It should be noted here that our previous work had not investigated the oxidation product of gold nanorods. ${ }^{40}$ Here we used mass spectrometry to identify the oxidation product. As shown in Fig. S3 in the ESI $\dagger$, the appearance of a peak at $\mathrm{m} / \mathrm{z}$ 925 indicated the formation of $\mathrm{AuBr}_{2}{ }^{-}-\left(\mathrm{CTA}^{+}\right)_{2}$ where $\mathrm{CTA}^{+}$is the abbreviation of cetyltrimethylammonium. The formation of $\mathrm{AuBr}_{2}{ }^{-}-\left(\mathrm{CTA}^{+}\right)_{2}$, an ionassociation compound, can be explained by the soft and hard acid-base theory that a cation with a large volume tends to associate with an anion with a large volume. ${ }^{43}$ According to our previous work and the mass spectrometry experimental results, we conclude that colorimetric sensing of the captured CuS is based on the following chemical equations.

$$
\begin{aligned}
\mathrm{CuS}+2 \mathrm{H}^{+} \rightarrow \mathrm{Cu}^{2+}+ & \mathrm{H}_{2} \mathrm{~S} \\
\mathrm{Cu}^{2+}+\mathrm{Au}+4 \mathrm{CTA}^{+}+4 \mathrm{Br}^{-} \rightarrow & \mathrm{CuBr}_{2}{ }^{-}-\left(\mathrm{CTA}^{+}\right)_{2} \\
& +\mathrm{AuBr}_{2}-\left(\mathrm{CTA}^{+}\right)_{2} \\
4 \mathrm{CuBr}_{2}{ }^{-}-\left(\mathrm{CTA}^{+}\right)_{2}+\mathrm{O}_{2}+4 \mathrm{H}^{+} \rightarrow & \mathrm{Cu}^{2+}+8 \mathrm{Br}^{-}+8 \mathrm{CTA}^{+} \\
& +2 \mathrm{H}_{2} \mathrm{O}
\end{aligned}
$$

Fig. 2 shows the LSPR absorption spectra and the colors of gold nanorods corresponding to different concentrations of human IgG. The longitudinal LSPR peak shifted to a short wavelength gradually with the increase in human IgG concentration in the range of $0.3-20 \mathrm{ng} \mathrm{ml}^{-1}$, accompanied by a color change from blue to purple and then to red. The LSPR peak-

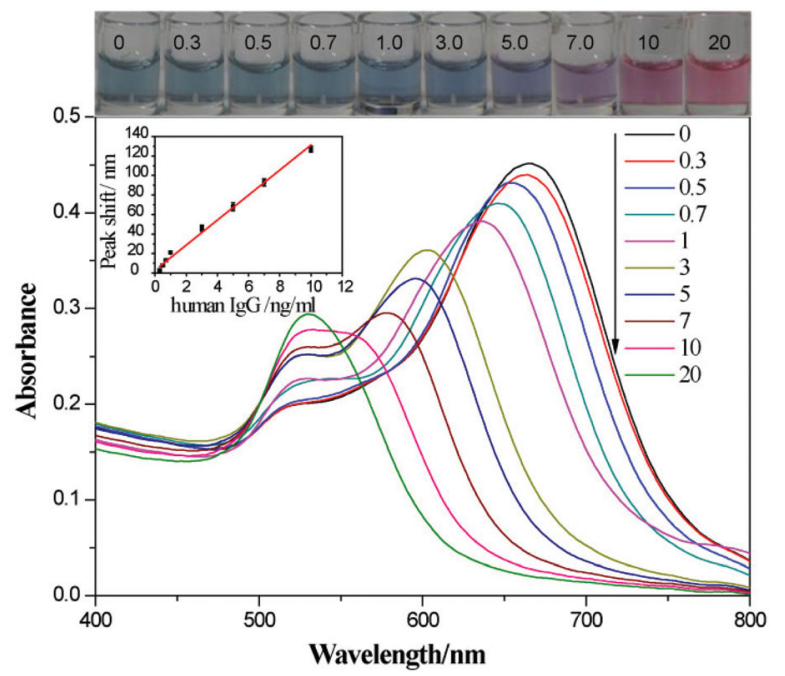

Fig. 2 LSPR absorption spectra and the colors of gold nanorods corresponding to different concentrations of human $\operatorname{lgG}\left(\mathrm{ng} \mathrm{ml}^{-1}\right)$. The inset is the longitudinal LSPR peak-shift vs. the concentration of human $\lg G\left(\mathrm{ng} \mathrm{ml}^{-1}\right)$. 
Table 1 Comparison of immunoassay methods developed for IgG

\begin{tabular}{|c|c|c|c|c|}
\hline Label & Immunoassay format & Analytical technique & Detection limit, $\mathrm{ng} \mathrm{ml^{-1 }}$ & Ref. \\
\hline HRP & ELISA & Spectrophotometry & 1.0 & 34 \\
\hline HPR & Immunomagnetic beads/sandwich & Spectrophotometry & 2.4 & 42 \\
\hline Ap & ELISA & Luminescence & 0.03 & 41 \\
\hline $\mathrm{Au}$ & Microwells/sandwich & Stripping voltammetry & 0.5 & 23 \\
\hline CdS & Immunomagnetic beads/sandwich & Stripping voltammetry & 10 & 24 \\
\hline $\mathrm{Au}$ & Capillary electrophoresis/homogeneous & Chemiluminescence & 1.14 & 25 \\
\hline Eu-BCPDA & Microwells/sandwich & Time-resolved fluorescence & 0.1 & 41 \\
\hline Pt & Microwells/sandwich & Spectrophotometry & 2.5 & 34 \\
\hline Si@Pt & Microwells/sandwich & Spectrophotometry & 10 & 31 \\
\hline $\mathrm{CuS}$ & Microwells/sandwich & Spectrophotometry & 0.15 & This work \\
\hline
\end{tabular}

shift could easily be monitored by using a simple microplate reader or a spectrophotometer. The detection limit was calculated to be $0.15 \mathrm{ng} \mathrm{ml^{-1 }}$ according to the $\mathrm{S} / \mathrm{N}=3$ rule which is lower than that of the traditional ELISA, HRP mimic nanomaterial tag-based immunoassay and electrochemical metalloimmunoassay and is comparable to the fluorescence immunoassay (Table 1). The digital photo (Fig. 2B) shows that

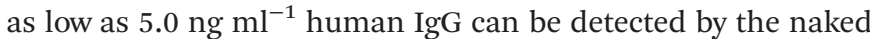
eye. The eye-readable color change also makes the metalloimmunoassay more convenient in clinical diagnosis, avoiding the use of complex equipment.

High tolerance to interferents and good specificity of the immunoassay can ensure the selective identification of the target analyte from the complex matrix. Fig. 3 shows the specificity and tolerance of the proposed immunoassay. The almost no obvious peak shift for the respective determination of $1.0 \mathrm{mg} \mathrm{m} \mathrm{m}^{-1}$ lactoglobulin, glucose, creatinine, glutamine, ascorbic acid, cysteine, catechin, papain, trypsin, peptone and

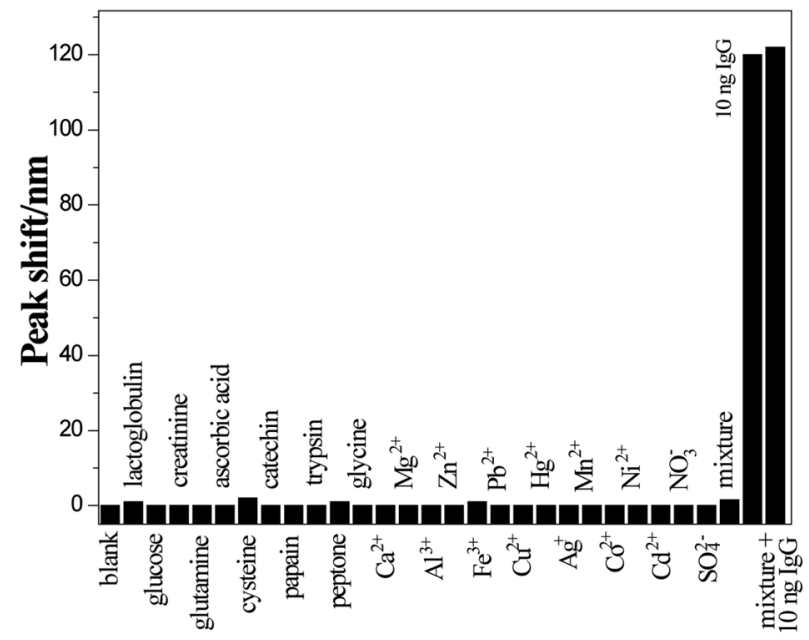

Fig. 3 LSPR peak shift of gold nanorods corresponding to $1.0 \mathrm{mg} \mathrm{m}^{-1}$ lactoglobulin, glucose, creatinine, glutamine, ascorbic acid, cysteine, catechin, papain, trypsin, peptone and glycine, and $1.0 \mu \mathrm{M} \mathrm{Ca}{ }^{2+}, \mathrm{Mg}^{2+}$, $\mathrm{Al}^{3+}, \mathrm{Zn}^{2+}, \mathrm{Fe}^{3+}, \mathrm{Pb}^{2+}, \mathrm{Cu}^{2+}, \mathrm{Hg}^{2+}, \mathrm{Ag}^{+}, \mathrm{Mn}^{2+}, \mathrm{Co}^{2+}, \mathrm{Ni}^{2+}, \mathrm{Cd}^{2+}, \mathrm{NO}_{3}^{-}$, $\mathrm{SO}_{4}{ }^{2-}$, the mixture of these chemicals, $10 \mathrm{ng} \mathrm{ml}^{-1}$ human IgG. glycine, and $1.0 \mu \mathrm{M} \mathrm{Ca}^{2+}, \mathrm{Mg}^{2+}, \mathrm{Al}^{3+}, \mathrm{Zn}^{2+}, \mathrm{Fe}^{3+}, \mathrm{Pb}^{2+}, \mathrm{Cu}^{2+}$, $\mathrm{Hg}^{2+}, \mathrm{Ag}^{+}, \mathrm{Mn}^{2+}, \mathrm{Co}^{2+}, \mathrm{Ni}^{2+}, \mathrm{Cd}^{2+}, \mathrm{NO}_{3}{ }^{-}, \mathrm{SO}_{4}{ }^{2-}$ and the mixture of these chemicals indicated that the metalloimmunoassay displayed good specificity. The almost the same change in the LSPR of gold nanorods for the determination of $10 \mathrm{ng} \mathrm{ml} \mathrm{m}^{-1}$ IgG in the presence and absence of the mixture of the above mentioned chemicals indicated that the proposed metalloimmunoassay possessed high tolerance to those chemicals.

Commonly, immunoassays are used to quantify target analytes in serum. An immunoassay with good specificity means the abundance of proteins, salts and other small molecules, such as glucose, ascorbic acid, etc., will do not interfere with the detection. Since the normal concentration of human IgG in human serum reaches $\mathrm{mg} \mathrm{mL}^{-1}$ levels, direct detection of human IgG in diluted human serum cannot evaluate the specificity of the proposed immunoassay. In consideration of the fact that the concentrations of proteins, salts and other small molecules in animal serum are comparable to human serum, here we choose the determination of spiked human IgG in fetal bovine serum (FBS) to investigate the specificity. The detection results are shown in Table 2 . The recoveries of the spiked human IgG ranged from $104.2 \%$ to $106.7 \%$. The satisfactory recoveries for determination of low concentrations of human IgG indicate that such a method has good specificity and is applicable to the quantification of low abundance proteins in complex biological samples, avoiding the interference from proteins, salts and other small molecules.

In conclusion, here we have developed a highly sensitive colorimetric metalloimmunoassay based on copper-mediated etching of gold nanorods. Since the cupric ion can accelerate the etching of gold nanorods by dissolved oxygen dramatically, the amount of captured CuS nanocrystals in a sandwich

Table 2 Determination results in the spiked sample by the proposed metalloimmunoassay

\begin{tabular}{llll}
\hline Samples & Added, $\mathrm{ng} \mathrm{ml}^{-1}$ & Found, $\mathrm{ng} \mathrm{ml}$ & Recovery \\
\hline FBS & 0.7 & $0.73 \pm 0.02$ & $104.3 \pm 2.8$ \\
& 3.0 & $3.2 \pm 0.1$ & $106.7 \pm 3.3$ \\
& 7.0 & $7.3 \pm 0.4$ & $104.2 \pm 5.7$
\end{tabular}


immunoreaction process can be monitored by using a simple microplate reader, spectrophotometer and even by the naked eye for the assay of the target analyte with relatively high concentrations, making the metalloimmunoassay easier and more convenient. The metalloimmunoassay is also more sensitive than the traditional ELISA, electrochemical immunoassay and HRP mimic nanomaterial tag-based immunoassay and comparable to the fluorescence immunoassay. The good specificity confirmed by the determination of human IgG in spiked samples indicates that the metalloimmunoassay is applicable to the quantification of the target analyte in complex samples. In the consideration of the pervasive application of immunoassays, the metalloimmunoassay promises to be an effective approach in clinical diagnosis and environmental analysis for detection of biomarkers and contaminants, such as heavy metal ions, pesticides and pathogenic bacteria.

This research was financially supported by the Strategic Priority Research Program of the Chinese Academy of Sciences (XDA11020702), the Department of Science and Technology of Shandong Province (BS2009DX006), NSFC (no. 21275158, 21175084, 21275091), CAS (KZCX2-YW-JS208) and the 100 Talents Program of the CAS.

\section{Notes and references}

1 Y. Zhang, H. Li, L.-Y. Niu, Q.-Z. Yang, Y.-F. Guan and L. Feng, Analyst, 2014, 139, 3146.

2 S.-X. Li, X. Lin, F.-Y. Zheng, W. Liang, Y. Zhong and J. Cai, Anal. Chem., 2014, 86, 7079.

3 B. Jiang, F. Li, C. Yang, J. Xie, Y. Xiang and R. Yuan, Anal. Chem., 2015, 87, 3094.

4 L.-Y. Niu, M.-Y. Jia, P.-Z. Chen, Y.-Z. Chen, Y. Zhang, L.-Z. Wu, C.-F. Duan, C.-H. Tung, Y.-F. Guan, L. Feng and Q.-Z. Yang, RSC Adv., 2015, 5, 13042.

5 H. Zhang, Y. Huang, S. Hu, Q. Huang, C. Wei, W. Zhang, L. Kang, Z. Huang and A. Hao, J. Mater. Chem. C, 2015, 3, 2093.

6 Q. Huang, X. Lin, C. Lin, Y. Zhang, S. Hu and C. Wei, RSC Adv., 2015, 5, 54102.

7 L. Feng, Y. Zhang, L. Wen, L. Chen, Z. Shen and Y. Guan, Analyst, 2011, 136, 4197.

8 S. Blumenthal, Perspect. Biol. Med., 2009, 52, 343.

9 R. Ekins and D. Kelso, Clin. Chem., 2011, 57, 372.

10 J. P. Gaut, D. L. Crimmins, M. F. Ohlendorf, C. M. Lockwood, T. A. Griest, N. A. Brada, M. Hoshi, B. Sato, R. S. Hotchkiss, S. Jain and J. H. Ladenson, Clin. Chem., 2014, 60, 747.

11 X. Liu, Q. Dai, L. Austin, J. Coutts, G. Knowles, J. Zou, H. Chen and Q. Huo, J. Am. Chem. Soc., 2008, 130, 2780.

12 S. D. Gan and K. R. Patel, J. Invest. Dermatol., 2013, 133, e12.

13 B. Nilsson, Curr. Opin. Immunol., 1989, 2, 898.

14 T. Porstmann and S. T. Kiessig, J. Immunol. Methods, 1992, 150, 5.

15 R. M. Lequin, Clin. Chem., 2005, 51, 2415.
16 J. Shen, Y. Li, H. Gu, F. Xia and X. Zuo, Chem. Rev., 2014, 114, 7631.

17 L. Z. Gao, J. M. Wu, S. Lyle, K. Zehr, L. L. Cao and D. Gao, J. Phys. Chem. C, 2008, 112, 17357.

18 J. D. Conradie, M. Govender and L. Visser, J. Immunol. Methods, 1983, 59, 289.

19 J. Tang and D. P. Tang, Microchim. Acta, 2015, 182, 2077.

20 S. L. Zhu, Y. Q. Fu and J. Z. Hou, J. Comput. Theor. Nanosci., 2010, 7, 1855.

21 N. L. Rosi and C. A. Mirkin, Chem. Rev., 2005, 105, 1547.

22 H. A. Ho, A. Najari and M. Leclerc, Acc. Chem. Res., 2008, 41, 168.

23 M. Dequaire, C. Degrand and B. Limoges, Anal. Chem., 2000, 72, 5521.

24 G. Liu, J. Wang, J. Kim, M. R. Jan and G. E. Collins, Anal. Chem., 2004, 76, 7126.

25 Y. M. Liu, L. Mei, L. J. Liu, L. F. Peng, Y. H. Chen and S. W. Ren, Anal. Chem., 2011, 83, 1137.

26 N. H. Tung, M. Chikae, Y. Ukita, P. H. Viet and Y. Takamura, Anal. Chem., 2012, 84, 1210.

27 Y. Wang, L. J. Tang and J. H. Jiang, Anal. Chem., 2013, 85, 9213.

28 W. He, Y. Liu, J. Yuan, J. J. Yin, X. Wu, X. Hu, K. Zhang, J. Liu, C. Chen, Y. Ji and Y. Guo, Biomaterials, 2011, 32, 1139.

29 Z. X. Zhang, Z. J. Wang, X. L. Wang and X. R. Yang, Sens. Actuators, B, 2010, 147, 428.

30 Z. Gao, M. Xu, M. Lu, G. Chen and D. Tang, Biosens. Bioelectron., 2015, 70, 194.

31 Z. Wang, X. Yang, J. Yang, Y. Jiang and N. He, Anal. Chim. Acta, 2015, 862, 53.

32 L. Gao, J. Zhuang, L. Nie, J. Zhang, Y. Zhang, N. Gu, T. Wang, J. Feng, D. Yang, S. Perrett and X. Yan, Nat. Nanotechnol., 2007, 2, 577.

33 Y. C. Lee, M. I. Kim, M. A. Woo, H. G. Park and J. I. Han, Biosens. Bioelectron., 2013, 42, 373.

34 Z. Gao, M. Xu, L. Hou, G. Chen and D. Tang, Anal. Chim. Acta, 2013, 776, 79.

35 L. Zhan, C. M. Li, W. B. Wu and C. Z. Huang, Chem. Commun., 2014, 50, 11526.

36 X. Que, D. Tang, B. Xia, M. Lu and D. Tang, Anal. Chim. Acta, 2014, 830, 42.

37 Y. Y. Chen, H. T. Chang, Y. C. Shiang, Y. L. Hung, C. K. Chiang and C. C. Huang, Anal. Chem., 2009, 81, 9433.

38 Z. Zhang, Z. Chen and L. Chen, Langmuir, 2015, 31, 9253.

39 Z. Zhang, Z. Chen, D. Pan and L. Chen, Langmuir, 2015, 31, 643.

40 Z. Zhang, Z. Chen, C. Qu and L. Chen, Langmuir, 2014, 30, 3625.

41 R. A. Evangelista, A. Pollak and E. F. G. Templeton, Anal. Biochem., 1991, 197, 213.

42 A. Ambrosi, M. T. Castaneda, A. J. Killard, M. R. Smyth, S. Alegret and A. Merkoci, Anal. Chem., 2007, 79, 5232.

43 J. Rodriguez-Fernandez, J. Perez-Juste, P. Mulvaney and L. M. Liz-Marzan, J. Phys. Chem. B, 2005, 109, 14257. 